\title{
Evaluación de la eficiencia de grupos de investigación mediante análisis envolvente de datos (DEA)
}

\author{
Por José-Luis Pino-Mejías, Francisco M. Solís-Cabrera, Mercedes Delgado-Fernández y Rosario Barea-Barrera
}

\begin{abstract}
Resumen: La evaluación de los resultados de los grupos de investigación en función de los recursos de que disponen y la obtención de indicadores de eficiencia constituyen cuestiones de gran relevancia para el diseño de las políticas de fomento de la investigación y el desarrollo $(I+D)$. Los profesionales de la información pueden contribuir a esta tarea mediante el suministro de datos sobre indicadores tales como el total de tesis doctorales o las publicaciones en las revistas de mayor prestigio. Se propone una metodología para la evaluación de la eficiencia técnica de los grupos de investigación basado en el análisis envolvente de datos (DEA) y se aplica a los grupos de investigación universitarios del área de tecnologías de la información y las comunicaciones (TIC) de Andalucía.
\end{abstract}

Palabras claves: Evaluación de la investigación, Análisis envolvente de datos (DEA), Medición de la ciencia, Eficiencia técnica, Indicadores de ciencia y tecnología.

Title: Assessing the efficiency of research groups using data envelopment analysis (DEA)

Abstract: Assessing the results of research groups based on available resources and obtaining some indicator of efficiency are issues of great relevance for the design of policies to promote research and development $(R \& D)$. Information professionals can contribute to this effort by providing data such as the total number of doctoral theses or publications in the most prestigious journals. The article proposes a methodology for assessing the technical efficiency of research groups based on data envelopment analysis (DEA) and applies it to university research groups in the area of information technology and communications (ICT) in Andalusia.

Keywords: Research assessment, Data envelopment analysis (DEA), Technical efficiency, Measurement of the science, Science and technology indicators.

Pino-Mejías, José-Luis; Solís-Cabrera, Francisco M.; Delgado-Fernández, Mercedes y Barea-Barrera, Rosario.

"Evaluación de la eficiencia de grupos de investigación mediante análisis envolvente de datos (DEA)". El profesional de la información, 2010, marzo-abril, v. 19, n. 2, pp. 160-167.

DOI: 10.3145/epi.2010.mar.06
José-Luis Pino-Mejías, es licenciado y doctor en matemáticas y profesor del Depto. de Estadística e Investigación Operativa de la Univ. de Sevilla. Fue DG de Universidades e Investigación de la Junta de Andalucía. Actualmente es secretario general del Centro Andaluz de Prospectiva, responsable del Grupo de Investigación "Métodos Cuantitativos en Evaluación" y director de proyectos sobre aplicación de la estadística y la investigación operativa a la gestión de empresas y administraciones.

Mercedes Delgado-Fernández, ingeniera industrial y doctora en ciencias técnicas del Instituto Superior Politécnico "José Antonio Echeverría" (Cujae) de La Habana, ejerce como decana de la Facultad de Ingeniería Industrial de esa universidad. Representante del Cujae en Eureka Iberoamérica. Se ha dedicado a la gestión de la I+D+i, participando desde hace 6 años en investigaciones relacionadas con la evaluación de la $I+D+i$ en la Universidad de Sevilla.

Rosario-del-Carmen Barea-Barrera, es licenciada en ciencias y técnicas estadísticas, siendo su línea de investigación actual el análisis envolvente de datos y los indicadores de ciencia y tecnología. Entre sus trabajos recientes está el capítulo "Descubrimiento de conocimiento en los indicadores de ciencia e innovación para el desarrollo sostenible" y el artículo "Evaluación integrada de la innovación, la tecnología y las competencias en la empresa".

Francisco-Manuel Solís-Cabrera, doctor en economía, licenciado en matemáticas y diplomado en empresariales por la Univ. de Sevilla, cuenta con numerosas publicaciones en materia de gestión, planificación y evaluación de la I+D+i, p. ej. "Indicadores bibliométricos de la producción científica de Andalucía 2003-2005". Es profesor titular en el Depto. de Economía Aplicada de la citada universidad. Ocupa la Secretaría del Plan Andaluz de Investigación, Desarrollo e Innovación de la Junta de Andalucía.

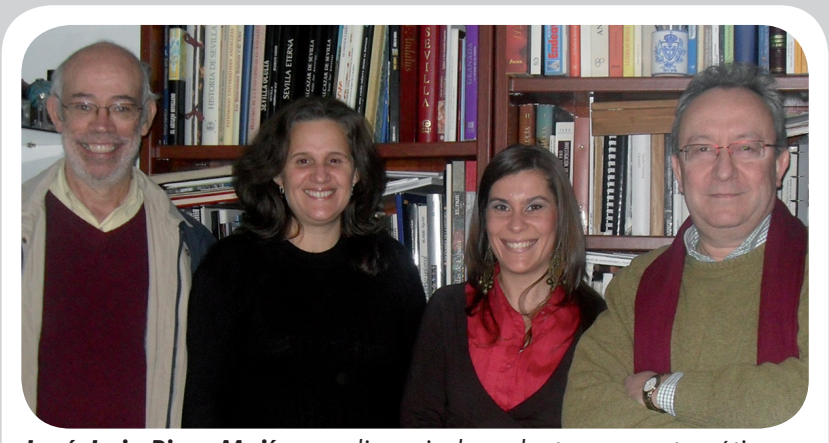

\section{Introducción}

El impacto social de la investigación científica y técnica ha sido en los últimos años objeto de estudio por parte de diversos colectivos, especialmente de la administración. El establecimiento de indicadores que midan este impacto desde diferentes perspectivas, de forma horizontal o sectorial, ha sido una preocupación constante para los responsables de diseñar y ejecutar las políticas científicas y tecnológicas no sólo a escala regional, nacional o internacional, sino también en ámbitos más restringidos como pueden ser las universidades y los grupos de investigación.

A nivel internacional se cuenta con manuales para la medición de las actividades de la ciencia, la tecno- 
logía y la innovación, como el Manual de Oslo, 2006; Manual de Frascati, 2003; Manual de Bogotá, 2001, y otros, que constituyen un marco homogéneo de referencia para la elaboración de las estadísticas oficiales. Sin embargo su uso ha estado limitado por la calidad y disponibilidad de la información existente. Evaluar la I + D+i desde el punto de vista de su impacto (Subramanian, 1996), de los efectos de sus gastos (Kostoff; Geisler, 2007), de las patentes y publicaciones que se generan (Nelson, 2009), y su situación a escala internacional es crucial para el diseño de las políticas públicas.

\section{"Ninguna técnica por sí sola es capaz de medir la eficiencia de la tarea que realizan los grupos de investigación"}

Los indicadores de ciencia e innovación reflejan el potencial de información científica técnica que se genera en un país. Las revistas científicas son el modo primario de comunicación formal en la ciencia (Togia; Tsigilis, 2006). La cantidad de trabajos publicados en revistas de prestigio o el número de patentes solicitadas $\mathrm{u}$ otorgadas son indicadores que miden el desempeño de los investigadores. Sin embargo son muchas las investigaciones que tratan de medir no sólo la producción científica, sino la eficiencia en el uso de los recursos por parte de los grupos de investigación. Se trata, por ejemplo, de explicar cuáles son los mecanismos gubernamentales que impulsan la investigación en las universidades (Rasmussen, 2008); cómo deben tomarse las decisiones para la asignación de recursos financieros para la innovación (Peneder, 2008); cómo gestionar la inversión en proyectos de tecnologías de información (Wu; Ong, 2008); cuál es la eficiencia productiva de la investigación en universidades (Cherchye; VandenAbeele, 2005); cómo analizar la influencia del tamaño de la institución o la concentración de investigadores en la productividad científica de los centros de investigación (Bonaccorsi; Daraio, 2005); o cómo medir las diferentes formas del capital intelectual con los procesos de producción del conocimiento y los resultados de las organizaciones de investigación (Leitner; Warden, 2004). En este trabajo se muestran las posibilidades del uso del Análisis Envolvente de Datos (DEA) para estos fines.

El DEA es una técnica que, a partir de datos sobre recursos empleados y resultados obtenidos para un conjunto de Unidades de Toma de Decisión (DMU), hace posible la evaluación de la eficiencia relativa de cada una de ellas.
Ejemplos de aplicación del DEA han sido: selección de proyectos de tecnologías de la información (Chen et al., 2006), medición de la eficiencia relativa de proyectos de I+D+i financiados por el gobierno de Taiwán (Hsu; Hsueh, 2009), evaluación de las actividades de I+D+i de diversos países (Wang; Huang, 2007), comparación de departamentos universitarios de la misma disciplina en el Reino Unido (Beasley, 1990), capacidad de adaptación estratégica de los institutos públicos de investigación italianos (Coccia, 2008), eficiencia de la I+D+i en la industria farmacéutica japonesa (Hashimoto; Shoko, 2008), o el examen de la eficiencia relativa en la investigación en 109 universidades chinas (Johnes; Yu, 2008).

Una primera conclusión de la revisión de estas aplicaciones es que los aspectos fundamentales son la selección de las variables de entrada y salida, y la calidad de la información disponible.

El Sistema de Información Científica de Andalucía $(S I C A)^{1}$ dispone de información con el nivel de desagregación y fiabilidad requerido para aplicar la técnica DEA, lo que aporta nuevos elementos de juicio a la administración y a los responsables en política científica en la toma de decisiones.

La implementación de un sistema de estas características en un dominio geográfico como Andalucía ${ }^{2}$ permitió evaluar las posibilidades y limitaciones de la metodología propuesta para su aplicación en otras regiones y países.

\section{"Los aspectos fundamentales son: la selección de las variables de entrada y salida, y la calidad de la información disponible"}

El presente trabajo muestra cómo puede emplearse el DEA para medir la eficiencia de los grupos de investigación e ilustra la metodología con su aplicación al área científico-técnica de las tecnologías de información y las comunicaciones (TIC) en Andalucía.

\section{Selección de unidades y variables}

La técnica DEA requiere que las DMUs seleccionadas cumplan las siguientes condiciones:

- Ser homogéneas para que se puedan comparar y lo suficientemente heterogéneas para que se pueda extraer información de la comparación (Charnes; Cooper; Rhodes, 1981). 
- Tener alguna capacidad para gestionar los recursos de que disponen.

- Ser su número suficiente para no limitar la capacidad discriminatoria del análisis.

El número de grupos de investigación para los que se dispone de información exhaustiva en el SICA supera los 1.800. A efectos de la gestión de las convocatorias de ayuda a la investigación estos grupos se integran en diversas áreas. Y de entre ellas, para ilustrar la aplicación de la técnica DEA, se ha optado por la de las tecnologías de la información y comunicaciones (TIC) en la que en 2009 había 86 grupos de investigación.

Seleccionadas las unidades para el análisis de eficiencia, el siguiente paso en la especificación del modelo es la selección de las variables de entradas y salidas, como aquellas capaces de representar de forma adecuada la actividad realizada.

En referencia a este proceso los autores que han llevado a cabo trabajos similares no dejan de señalar la gran complejidad que conlleva esta etapa y más, al tratarse del entorno universitario (Anderson; Tugrul; Francois, 2007) en el que el fin primordial es la creación y difusión del conocimiento.

Existen diferentes enfoques para la medición de la ciencia, entre ellos el basado en la bibliometría o el de los indicadores de I+D+i. Estos enfoques no son independientes entre sí, pero las diferencias en los datos y las técnicas que se emplean en cada uno de ellos provocan que los resultados no sean comparables.

En los trabajos recientemente publicados se observa que como variables de entrada suelen elegirse los gastos de $\mathrm{I}+\mathrm{D}+\mathrm{i}$, el número de investigadores, técnicos y personal de apoyo y como variables de salida las patentes y las publicaciones científicas (Wang; Huang, 2007), (Meng et al., 2008), (Groot; García-Valderrama, 2006), (Thursby; Kemp, 2002), (Delgado-Fernández et al., 2008).

En España, los grupos de investigación reciben fundamentalmente financiación pública de los planes de $\mathrm{I}+\mathrm{D}+\mathrm{i}$ regionales, nacionales y europeos.

\section{"La existencia del Sistema de Información Científica de Andalucía permite la utilización de la técnica DEA para la medición de la eficiencia de los grupos de investigación andaluces"}

En Andalucía uno de los fines explícitos de las ayudas regionales a los grupos de investigación es incre- mentar la participación de los grupos andaluces en los programas nacionales e internacionales, de ahí que a efectos de esta política de incentivos se consideren las ayudas del gobierno andaluz como una entrada y las que se obtienen del resto de programas públicos, y los contratos con empresas, como un resultado de la actividad de los grupos. Los criterios para la evaluación de las ayudas al funcionamiento anual de los grupos de investigación están establecidos en el artículo 7 de la Orden de la Consejería de Innovación, Ciencia y Empresa de la Junta de Andalucía de 3 de septiembre de 2007 (BOJA de 21 de septiembre de 2007), por la que se regula el funcionamiento del Registro electrónico de agentes del sistema andaluz del conocimiento para los grupos de investigación dependientes de las universidades y organismos de investigación ubicados en Andalucía y se establece su financiación.

Desde esta perspectiva las variables que se han considerado inicialmente han sido las que se muestran en la tabla 1.

Este conjunto de variables es demasiado amplio para su inclusión en un modelo DEA, por ello se ha aplicado el análisis factorial como técnica de reducción de la dimensionalidad a los conjuntos de variables de entrada y salida, y la regresión lineal y la correlación canónica para identificar las relaciones entre las variables de entrada y salida, empleando el paquete estadístico SPSS 16 (Barea, 2009). Como resultado de la aplicación de estas técnicas las variables seleccionadas han sido las que se muestran en la tabla 2.

Los valores de estas variables en el período 20042007 para los grupos de investigación universitarios y del CSIC en Andalucía del área de las TIC figuran en la tabla 3.

En la categoría "Resto de grupos" se incluyen los datos de las Universidades de Almería, Cádiz, Córdoba, Pablo de Olavide y del Consejo Superior de Investigaciones Científicas en Andalucía. Cada una de estas instituciones tiene menos de diez grupos de investigación en el área de las TIC.

Para la determinación de la adecuación entre el número total de variables (n) y de unidades (DMU $=86)$ se aplican algunas reglas propuestas por varios autores; en nuestro caso se cumplen todas, al ser $\mathrm{p}=3$ el número de variables de entradas y $\mathrm{q}=5$ el de salidas.

Golany y Roll (1989):

$$
\mathrm{n} \geq 2 \times(\mathrm{p}+\mathrm{q})=16 ; \quad 86 \geq 16
$$

Charnes, Cooper y Rhodes (1994):

$\mathrm{n} \geq 3 \times(\mathrm{p}+\mathrm{q})=24$

$86 \geq 24$

Murias-Fernández (2004):

$\mathrm{n} \geq \mathrm{p} \times \mathrm{q}=15 ;$

$86 \geq 15$ 


\begin{tabular}{|c|c|}
\hline Entradas (Input) & Salidas (Output) \\
\hline Recursos humanos & Publicaciones \\
\hline $\begin{array}{l}\text { Número de doctores } \\
\text { activos }\end{array}$ & Revistas ISI \\
\hline $\begin{array}{l}\text { Número de titulados } \\
\text { superiores }\end{array}$ & Revistas UCUA \\
\hline \begin{tabular}{|l|} 
Número de titulados \\
medios
\end{tabular} & Otras revistas \\
\hline Personal auxiliar & Libros \\
\hline Becarios & Capítulos de libros \\
\hline Recursos financieros & Aportaciones a congresos \\
\hline \multirow{3}{*}{$\begin{array}{l}\text { Subvenciones recibidas } \\
\text { en la convocatoria de } \\
\text { ayudas a los grupos de } \\
\text { investigación }\end{array}$} & $\begin{array}{l}\text { Aportaciones a congresos } \\
\text { internacionales }\end{array}$ \\
\hline & $\begin{array}{l}\text { Aportaciones a congresos } \\
\text { nacionales }\end{array}$ \\
\hline & Comunicaciones en congresos \\
\hline & Otras aportaciones a congresos \\
\hline & Tesis \\
\hline & Tesis realizadas \\
\hline & Tesis dirigidas \\
\hline & Total tesis \\
\hline & Propiedad industrial \\
\hline & Patentes en explotación \\
\hline & Otras patentes \\
\hline & Total patentes \\
\hline & Otros tipos innovación $(*)$ \\
\hline & Total propiedad industrial \\
\hline & Proyectos \\
\hline & $\begin{array}{l}\text { Proyectos financiación pública } \\
\text { externa dirigidos }\end{array}$ \\
\hline & $\begin{array}{l}\text { Total proyectos financiación } \\
\text { pública externa }\end{array}$ \\
\hline & Otros proyectos participados $(* *)$ \\
\hline & Otros proyectos dirigidos $(* * *)$ \\
\hline & Total otros proyectos \\
\hline & Total proyectos \\
\hline & $\begin{array}{l}\text { Ingresos proyectos financiación } \\
\text { pública }\end{array}$ \\
\hline & Contratos \\
\hline & Contratos LOU \\
\hline & Otros contratos \\
\hline & Total contratos \\
\hline & Otras actividades \\
\hline & Comités de revistas \\
\hline & Trabajos creativos \\
\hline & Resultados relevantes \\
\hline & Total otras actividades \\
\hline
\end{tabular}

$I S I=\quad$ Institute for Scientific Information, Thomson Reuters

UCUA = Unidad para la Calidad de las Universidades Andaluzas

(*) Los que no están recogidos en los ítems anteriores

(**) En los que el grupo ha participado no imputados en los ítems anteriores

(***) Dirigidos por el grupo no imputados en los ítems anteriores

Tabla 1

\begin{tabular}{|c|c|}
\hline Entradas (Input) & Salidas (Output) \\
\hline Número de doctores activos & $\begin{array}{l}\text { Ingresos por proyectos de } \\
\text { financiación pública }\end{array}$ \\
\hline Resto de personal de I+D+i (*) & Publicación en revistas ISI \\
\hline \multirow{3}{*}{$\begin{array}{l}\text { Subvenciones recibidas en la } \\
\text { convocatoria de ayudas a los } \\
\text { grupos de investigación }\end{array}$} & $\begin{array}{l}\text { Número de tesis total } \\
(* *)\end{array}$ \\
\hline & Total otros proyectos \\
\hline & Número de contratos total \\
\hline \multicolumn{2}{|c|}{$\begin{array}{l}\text { Número de titulados superiores + Número de titulados medios + Personal auxi } \\
\text { liar + Becarios } \\
\text { Número de tesis realizadas + Número de tesis dirigidas }\end{array}$} \\
\hline
\end{tabular}

Tabla 2

Así, pues, el número de variables de entradas y de salidas seleccionadas cumplen los requisitos para medir la eficiencia técnica mediante la metodología DEA.

\section{Aplicación del DEA}

El propósito final de la aplicación del DEA es responder a las cuestiones relativas a: ¿cuáles son las unidades eficientes e ineficientes?, ¿cuáles son los grupos de referencias? y ¿cuáles son las mejoras potenciales que deben realizar las unidades menos eficientes para alcanzar la eficiencia? Contestar a estas cuestiones conlleva la construcción de un modelo matemático de eficiencia, pero antes se debe especificar la orientación del análisis que se va a ejecutar, es decir, dar a conocer si lo que se pretende en la investigación es la obtención de resultados que busquen la maximización de las salidas (outputs) o, por el contrario, la minimización de las entradas (inputs).

\section{"¿Cuáles son las mejoras potenciales que deben realizar las unidades menos eficientes para alcanzar la eficiencia?"}

En esta investigación se quiere analizar la eficiencia mostrada por los grupos de investigación en su labor científica. Por ello la primera orientación (máximo output) es la más coherente.

Sin profundizar en los fundamentos matemáticos de la técnica DEA, podemos señalar que el modelo de eficiencia consiste en la optimización del cociente entre la suma ponderada de salidas y la suma ponderada de entradas, sujeto lógicamente a la restricción de que este cociente sea igual o menor que 1 para todas las unidades consideradas.

En este punto es necesario concretar y definir si se optará por la utilización de un modelo con rendimientos de escala constante (CCR), o uno con rendimientos 


\begin{tabular}{|c|c|c|c|c|c|}
\hline & $\begin{array}{l}\text { Univ. } \\
\text { Granada }\end{array}$ & $\begin{array}{l}\text { Univ. } \\
\text { Málaga }\end{array}$ & $\begin{array}{l}\text { Univ. } \\
\text { Sevilla }\end{array}$ & $\begin{array}{c}\text { Resto } \\
\text { de grupos }\end{array}$ & Total \\
\hline Número de grupos (DMUs) & 22 & 14 & 20 & 30 & 86 \\
\hline \multicolumn{6}{|l|}{ Variables de entrada } \\
\hline Número de doctores activos & 189 & 168 & 146 & 190 & 693 \\
\hline Resto de personal de $\mathrm{I}+\mathrm{D}+\mathrm{i}$ & 59 & 65 & 74 & 57 & 255 \\
\hline $\begin{array}{l}\text { Subvenciones recibidas en la convocatoria } \\
\text { de ayudas a los grupos de investigación }\end{array}$ & 256.719 & 287.295 & 201.752 & 256.161 & 1.001 .926 \\
\hline \multicolumn{6}{|l|}{ Variables de salida } \\
\hline $\begin{array}{l}\text { Ingresos por proyectos de financiación } \\
\text { pública }\end{array}$ & 13.628 .998 & 26.298 .098 & 12.176 .814 & 12.484 .676 & 64.588 .585 \\
\hline Publicación en revistas ISI & 588 & 450 & 458 & 896 & 2.392 \\
\hline Total otros proyectos & 96 & 46 & 48 & 80 & 270 \\
\hline Número de contratos total & 85 & 144 & 221 & 132 & 582 \\
\hline
\end{tabular}

Tabla 3. Totales por instituciones

de escala variable (BCC). Un método para averiguar si es mejor usar uno u otro, es dibujar los gráficos de dispersión de los pares formados al elegir una variable de entrada y otra de salida. En la figura 1 se observa que los puntos frontera se ajustan a una función lineal; por tanto, es aconsejable utilizar el modelo CCR.

Una vez definido el modelo se procede a su resolución. Para ello se ha utilizado el software Cplex.

Respecto a los resultados que se han obtenido (Barea-Barrera, 2009), se observa la existencia de grupos $100 \%$ eficientes, tal como muestra la figura 2 (19 unidades $100 \%$ eficientes), así como 31 unidades cuyo grado de eficiencia varía entre el $60 \%$ y el $99 \%$. No obstante, existen 36 grupos de investigación muy ineficientes, no llegando ni al $60 \%$.

Según la técnica DEA, las unidades eficientes obtienen el máximo rendimiento de los recursos de que disponen, en cambio las unidades menos eficientes pueden conocer qué deberían lograr para alcanzar el mismo nivel de eficiencia que las mejores unidades.

La técnica DEA crea grupos de referencia, de forma que cada unidad no eficiente es comparada con un

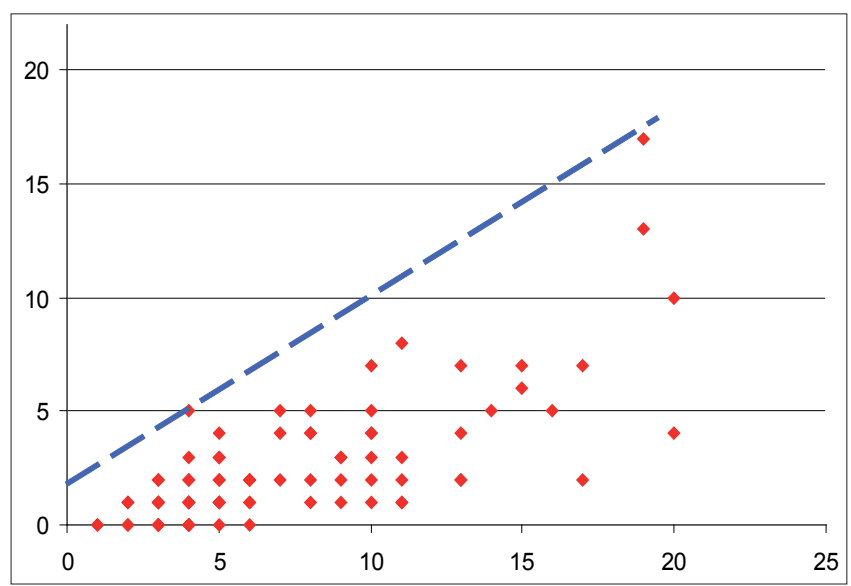

Figura 1. Gráfico de dispersión de las variables № de doctores activos y № total de tesis. grupo de unidades con recursos semejantes y mejores resultados. Puede darse el caso de que una unidad $100 \%$ eficiente forme parte del grupo de referencia de más de una unidad ineficiente. En la figura 3 se muestra

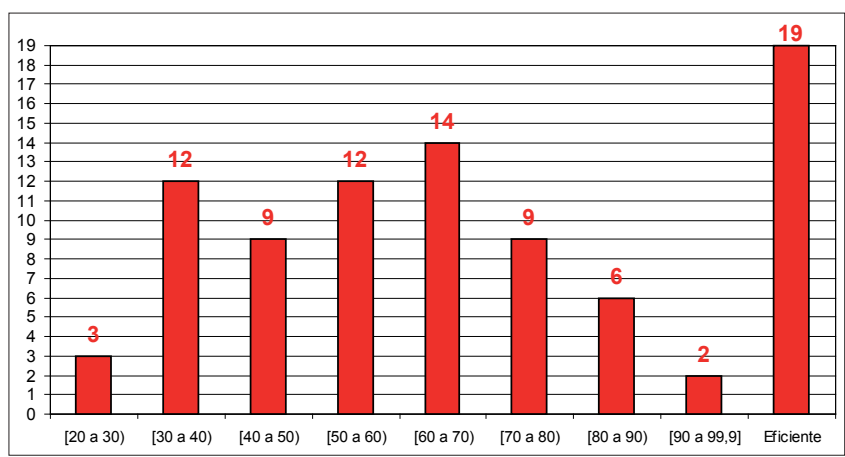

Figura 2. Distribución de resultados de eficiencia de los grupos de investigación TIC

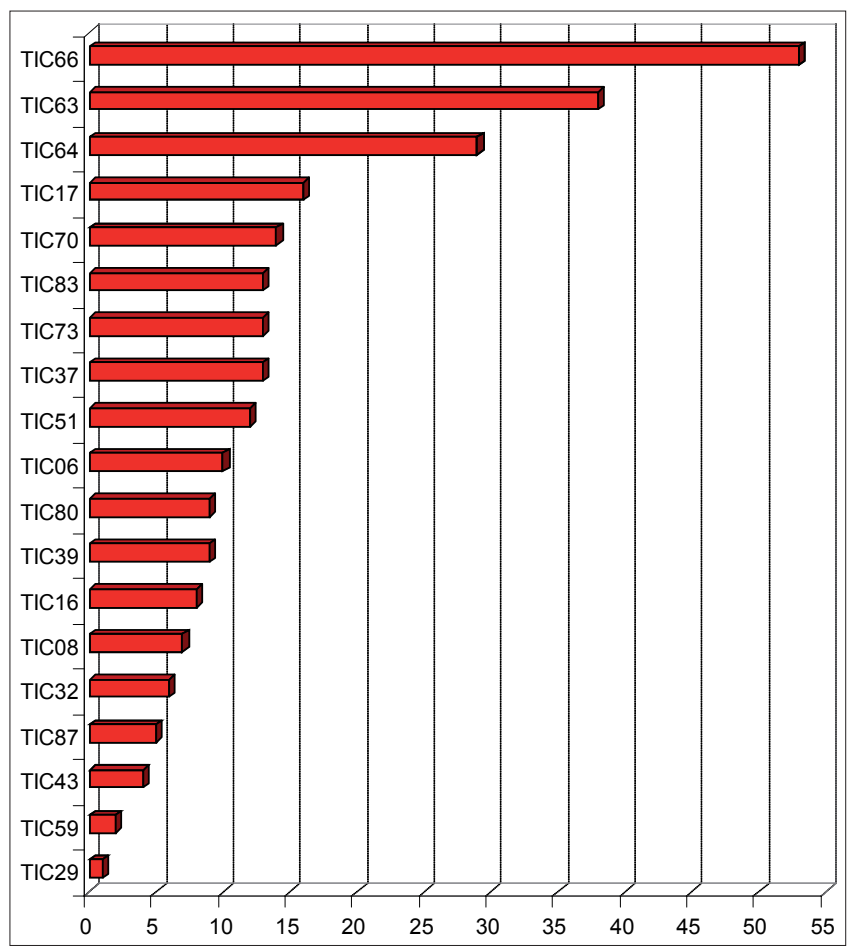

Figura 3. Frecuencia de grupos de referencia 
el número de veces que una unidad eficiente ha tomado parte del grupo de referencia de una no eficiente. Una frecuencia mayor en los grupos de referencia implica un mejor desempeño de esa unidad frente al resto de unidades eficientes. La unidad que aparece en un mayor número de grupos de referencia puede considerarse como líder global (con el fin de preservar el secreto estadístico, en este estudio las etiquetas de los grupos se han asignado al azar).

\section{“EI análisis muestra las mejoras potenciales que deben llevarse a cabo entre los grupos menos eficientes"}

Se observa que el grupo de investigación TIC66 es el líder global, al formar parte de los grupos de referencia de un mayor número de grupos de investigación no eficientes, exactamente en 53 ocasiones. Tampoco se quedan a mucha distancia los grupos de investigación TIC63 y TIC64, pues forman parte de hasta 38 y 29 grupos de referencia de grupos de investigación no eficientes respectivamente. El resto de grupos de investigación se encuentran presentes entre 1 y 16 grupos de referencia. El análisis de eficiencia no debe acabar en ese punto, sino que necesita ir un poco más allá y enseñar qué deberían hacer las unidades menos eficientes para alcanzar la frontera de eficiencia y así llegar a ser unidades $100 \%$ eficientes, es decir, mostrar cuáles son las mejoras potenciales que deben llevarse a cabo entre los grupos de investigación menos eficientes.

El propósito del análisis de las mejoras potenciales es indicar cuánto y en qué factores una unidad ineficiente debe mejorar para alcanzar la eficiencia. Esta información permite establecer objetivos que podrían guiar la mejora del desempeño de las unidades menos eficientes. De forma ilustrativa se muestra la mejora potencial de la unidad TIC45. Lo primero que se debe obtener es el conjunto de referencia de la unidad TIC45 (tabla 4).

Esta tabla significa que, para que TIC45 llegue a ser eficiente, se debe guiar de las unidades TIC39, TIC66,

\begin{tabular}{|c|c|}
\hline \multicolumn{1}{|c|}{ Unidad } & Eficiencia \\
\hline TIC39 & 0,789040 \\
\hline TIC66 & 0,542262 \\
\hline TIC70 & 0,013008 \\
\hline TIC83 & 0,249909 \\
\hline
\end{tabular}

Tabla 4. Conjunto de referencia del grupo TIC45
TIC70 y TIC83. Una vez que se tiene el conjunto de referencia el siguiente paso es determinar los valores objetivos, es decir, los valores que las variables deben tomar para llegar a ser eficientes (tabla 5).

La mejora potencial que debe lograr el grupo de investigación TIC45, se recoge en la tercera columna de la tabla 3. Se observa que debería aumentar en 21.635 euros los ingresos por proyectos de financiación pública, deberían lograr 7 publicaciones más en revistas ISI, el número de tesis total no necesita aumentar, y el total de otros proyectos y el número de contratos total deberían aumentar en 3 y en 1 respectivamente para que la unidad TIC45 llegara a ser plenamente eficiente.

\section{Conclusiones}

Aunque ninguna técnica por sí sola es capaz de medir la eficiencia de la tarea que realizan los grupos de investigación, la aplicación del DEA permite obtener un indicador sintético de la eficiencia técnica para cada grupo de investigación, ofrece la oportunidad de identificar las mejores prácticas y suministra valores de referencia que pueden servir de guía en los procesos de mejora.

La metodología expuesta aporta, en relación con otras aplicaciones del DEA a la evaluación de la eficiencia de centros o instituciones de investigación, la posibilidad de descender hasta el nivel de los grupos de investigación, al considerarlos como unidades de decisión de la actividad de $\mathrm{I}+\mathrm{D}+\mathrm{i}$.

Para que sea posible aplicar este tipo de técnicas es necesario disponer de sistemas de información científica que suministren información con un gran nivel de desagregación y fiabilidad.

\begin{tabular}{|l|r|r|r|}
\hline \multicolumn{1}{|c|}{ Input/Output } & $\begin{array}{c}\text { Valor } \\
\text { observado }\end{array}$ & $\begin{array}{c}\text { Valor } \\
\text { objetivo }\end{array}$ & $\begin{array}{c}\text { Mejora } \\
\text { potencial }\end{array}$ \\
\hline $\begin{array}{l}\text { Número de } \\
\text { doctores activos }\end{array}$ & 5 & 5 & 0 \\
\hline $\begin{array}{l}\text { Resto de personal } \\
\text { de I+D+i }\end{array}$ & 0 & 0 & 0 \\
\hline $\begin{array}{l}\text { Subvenciones } \\
\text { recibidas por } \\
\text { los grupos de } \\
\text { investigación }\end{array}$ & 5.109 & 5.109 & 0 \\
\hline $\begin{array}{l}\text { Ingresos por } \\
\text { proyectos de } \\
\text { financiación } \\
\text { pública }\end{array}$ & 559.607 & 581.242 & 21.635 \\
\hline $\begin{array}{l}\text { Publicación en } \\
\text { revistas ISI }\end{array}$ & 1 & 10 & 7 \\
\hline $\begin{array}{l}\text { Número de tesis } \\
\text { total }\end{array}$ & 2 & 1 & 0 \\
\hline $\begin{array}{l}\text { Total otros } \\
\text { proyectos }\end{array}$ & 17 & 18 & 3 \\
\hline $\begin{array}{l}\text { Número de } \\
\text { contratos total }\end{array}$ & 3 & 5 & 1 \\
\hline
\end{tabular}

Tabla 5. Valores observados, objetivos y mejora potencial de la unidad TIC45 
La existencia del Sistema de Información Científica de Andalucía permite la utilización de la técnica DEA para la medición de la eficiencia de los grupos de investigación andaluces. La aplicación a los 86 grupos andaluces del área TIC ha mostrado cómo la mayoría de ellos presentan niveles de eficiencia medios, existiendo un total de 19 grupos de investigación que son $100 \%$ eficientes. Se ha obtenido cuál ha sido el grupo de investigación líder, es decir, el que aparece como referencia en un número mayor de veces para las unidades ineficientes. Y por último, y de forma ilustrativa, se han identificado los resultados que debería lograr un grupo de investigación para llegar a ser plenamente eficiente, tomando como referencia los resultados de los grupos identificados como más eficientes.

\section{Notas}

1. El SICA fue creado en 2001 con el fin de adquirir, almacenar, organizar y recuperar la información relacionada con la actividad científico-técnica de los investigadores adscritos a centros de investigación, instituciones y universidades andaluzas.

2. Andalucía representa el $17,3 \%$ del territorio español. Es la comunidad autónoma más habitada de España, con el 17,8\% de los habitantes. Cuenta con 10 universidades públicas y 21.000 personas dedicadas a I+D+i en el sector público, de las cuales 12.000 son doctores.

\section{Referencias bibliográficas}

Albornoz, Mario et al. El estado de la ciencia. Principales indicadores de ciencia y tecnología iberoamericanos/interamericanos. Redes y Cyted, Buenos Aires, 2006.

Anderson, Timothy R.; Tugrul, U. Daim; Francois, F. Lavoie. "Measuring the efficiency of university technology transfer". Technovation, 2007, v. 27, n. 5, pp. 306-318.

Barea-Barrera, Rosario. Evaluación de la eficiencia mediante el análisis envolvente de datos. Trabajo de investigación, dirigido por José-Luis PinoMejía. Universidad de Sevilla, junio 2009.

Beasley, John E. "Comparing university departments". Omega, 1990, v. 18, n. 2, pp. 171-183.

Bonaccorsi, Andrea; Daraio, Cinzia. "Exploring size and agglomeration effects on public research productivity". Scientometrics, 2005, v. 63, n. 1, pp. $87-120$.

Cherchye, Laurens; Abeele, Piet Vanden. "On research efficiency: A micro-analysis of Dutch university research in economics and business management". Research policy, 2005, n. 34, pp. 495-516.

http://ideas.repec.org/e/pch127.html

Cervera, José-Luis. "Estadísticas e indicadores de ciencia, tecnología e innovación en América Latina y el Caribe". Comisión Económica para América Latina y el Caribe (Cepal). En: Primera reunión de la Conferencia estadística de las Américas de la Comisión Económica para América Latina y el Caribe. Santiago de Chile, 9-11 de mayo de 2001.

Chakrabarti, Alok K. "Technology indicators: conceptual issues and measurement problems". Journal of engineering and technology management, 1989, v. 6, n. 2, pp. 99-116.

Charnes, Abraham; Cooper, William W.; Lewin, Arie Y.; Seiford, Lawrence M. Data envelopment analysis: theory, methodology and applications. Springer, 1995. ISBN: 978-0-7923-9480-8.

Charnes, Abraham; Cooper, William W.; Rhodes, Edward. "Measuring the efficiency of decision making units". European journal of operational research, 1978, v. 2, n. 6, pp. 429-444.

Charnes, Abraham; Cooper, William W.; Rhodes, Edward. "Evaluating program and managerial efficiency: an application of data envelopment analysis to program follow through". Management science, 1981, v. 27, n. 6, pp. 668-697.

Chen, Hsing Hung; H. I. Lee, Amy; Tong, Yunhuan. "Analysis of new products mix selection at TFT-LCD technological conglomerate network under uncertainty". Technovation, 2006, v. 26, n. 11, pp. 1210-1221.

Chen, Yao; Liang, Liang; Yang, Feng; Zhu, Joe. "Evaluation of information technology investment: a data envelopment analysis approach". Computers and operation research, 2006, v. 33, n. 5, pp. 1368-1379.

Cherchye, Laurens; Vanden-Abeele, Piet. "On research efficiency: a micro-analysis of Dutch university research in economics and business management". Research policy, 2005, v. 34, n. 4, pp. 495-516.

Coccia, Mario. "Measuring scientific performance of public research units for strategic change". Journal of informetrics, 2008, v. 2, n. 3, pp. 183-194.

Comisión de las Comunidades Europeas. "Comunicación de la Comisión al Consejo y al Parlamento Europeo. La innovación en una economía del conocimiento". COM567 final. Bruselas, 2000.

Cplex

http://www.ilog.com/products/cplex/

Delgado-Fernández, Mercedes; Pino-Mejía, José-Luis; Solís-Cabrera, Francisco-Manuel; Barea-Barrera, Rosario. "Evaluación integrada, innovación, tecnología y competitividad". En: XI Seminario Latino Iberoamericano de gestión tecnológica Altec, 2007, 26-28 de septiembre, Buenos Aires.

Delgado-Fernández, Mercedes; Pino-Mejía, José-Luis; Solís-Cabrera, Francisco-Manuel; Barea-Barrera, Rosario. "Evaluación integrada de la innovación, la tecnología y las competencias en la empresa". Revista de $I+D+i, m i+d, 2008,47$.

http://www.madrimasd.org/revista/revista47/tribuna/tribunal.asp

Delgado-Fernández, Mercedes; Pino-Mejía, José-Luis; Solís-Cabrera, Francisco-Manuel; Barea-Barrera, Rosario. "Descubriendo el conocimiento en la innovación". En: XIV Congreso Latino Ibero-americano de Investigación de Operaciones (Claio), 2008, 2-9 de septiembre, Cartagena de Indias, Colombia.

Golany, Boaz; Roll, Yaakov. "An application procedure for DEA". Omega, 1989, v. 17, n. 3, pp. 237-250.

Gopalakrishnan, Shanthi; Bierly, Paul. "Analyzing innovation adoption using a knowledge-based approach". Journal of engineering and technology management, 2001, v. 18, n. 2, pp. 107-130.

Groot, Tom; García-Valderrama, Teresa. "Research quality and efficiency: an analysis of assessment and management issues in Dutch economic and business research programs". Research policy, 2006, v. 35, n. 9, pp. 1362-1376.

Grupp, Hariolf. "Efficiency of government intervention in technical change in telecommunications: ten national economies compared". Technovation, 1993, v. 13, n. 4, pp. 187-220.

Hair, Joseph F.; Anderson, Rolph E.; Tatham, Ronald L.; Black, William C. Análisis multivariante. Madrid: Prentice-Hall, 1999, 832. ISBN: 84-8322-035-0

Hashimoto, Akihiro; Shoko, Haneda. "Measuring the change in R\&D efficiency of the Japanese pharmaceutical industry". Research policy, 2008, v. 37, n. 10, pp. 1829-1836.

Hobday, Michael. "Evaluating collaborative R\&D programmes in information technology: the case of the UK Alvey programme". Technovation, 1998 , v. 8 , n. 4, pp. 271-298.

Hsu, Fang-Ming; Hsueh, Chao-Chih. "Measuring relative efficiency of government-sponsored R\&D projects: a three-stage approach". Evaluation and program planning, 2009, v. 32, n. 2, pp. 178-186.

Johnes, Jill; Yu, Li. "Measuring the research performance of Chinese higher education institutions using data envelopment analysis". China economic review, 2008, v. 19, n. 4, pp. 679-696.

Kostoff, Ronald; Geisler, Elie. "The unintended consequences of metrics in technology evaluation". Journal of informetrics, 2007, v. 1, n. 2, pp. 103-114.

Leitner, Karl-Heinz; Warden, Campbell. "Managing and reporting knowledge-based resources and processes in research organisations: specif- 
ics, lessons learned and perspectives". Management accounting research, 2004 , v. 15 , n. 1 , pp. $33-51$

Manual de Bogotá. Normalización de indicadores de innovación tecnológica en América Latina y el Caribe. Ricyt, OEA, Cyted/Colciencias, OCYT, Colombia, 2001.

Manual de Frascati. Propuesta de norma práctica para encuestas de investigación y desarrollo experimental. Fundación Española Ciencia y Técnica (Fecyt), 2003, ISBN 84-688-2888-2-OCDE.

Manual de OSLO. Guía para la recogida e interpretación de datos sobre innovación. OCDE y Eurostat, 2006.

Meng, Wei; Daqun, Zhang; Li, Qi; Wenbin, Lu. "Two-level DEA approaches in research evaluation”. Omega, 2008, v. 36, n. 6, pp. 950-957.

Murias-Fernández, Pilar. Metodología de aplicación del análisis envolvente de datos: evaluación de la eficiencia técnica en la Universidad de Santiago de Compostela, tesis doctoral, Universidad de Santiago de Compostela, 2004.

Nelson, Andrew J. "Measuring knowledge spillovers: what patents, licenses and publications reveal about innovation diffusion". Research policy, 2009, v. 38, n. 6, pp. 994-1005.

Parthasarthy, Raghavan; Hammond, Jan. "Product innovation input and outcome: moderating effects of the innovation process". Journal of engineering and technology management, 2002, v. 19, n. 1, pp. 75-91.

Peneder, Michael. "The problem of private under-investment in innovation a policy mind map". Technovation, 2008, v. 28, n. 2, pp. 518-530.

Rasmussen, Einar. "Government instruments to support the commercialization of university research: lessons from Canada". Technovation, 2008, v 28 , n. 8, pp. 506-517.

Red de Indicadores de Ciencia y Tecnología (Ricyt). http://www.ricyt.edu.ar/

Rondón, Leonor. "Indicadores del impacto de la ciencia y la tecnología (CT) en la sociedad: reflexiones y avances". Espacios, 2004, v. 25, n. 2. http://www.revistaespacios.com/a04v25n02/04250221.html

Sancho-Lozano, Rosa. Indicadores de los sistemas de ciencia, tecnología e innovación. Economía industrial, 2002, n. 343.

http://digital.csic.es/bitstream/10261/11958/1/097-SANCHO.pdf

Sinuany-Stern, Zilla; Abraham, Mehrez; Barboy, Arieh. Academic departments efficiency via DEA. Computers operation research, 1994, v. 21, n. 5 , pp. 543-556.

Solís-Cabrera, Francisco-Manuel. "El Sistema de Información Científica de Andalucía (SICA), una experiencia pionera en España”. Revista Mi+d. Monográfico Las Comunidades Autónomas frente a la I+D, 2008, n. 22, pp. 12-18.

http://www.madrimasd.org/informacionidi/revistas/monograficos/ monografias/monografia22/las_CA_frente_IDi-sistema_informacion_ cientifico_andalucia.pdf
SPSS.

http://www.spss.com/es/

Subramanian, Ashok. "Innovativeness: redefining the concept". Journal of engineering and technology management, 1996, v. 13, n. 3-4, pp. 223-243.

Thursby, Jerry; Kemp, Sucanya. "Growth and productive efficiency of university intellectual property licensing". Research policy, 2002, v. 31, n. 1, pp. 109-124.

Togia, Aspasia; Tsigilis, Nikolaos. "Impact factor and education journals: a critical examination and analysis". International journal of educational research, 2006, v. 45, n. 6, pp. 362-379.

Van Raan, Anthony F. "Evaluation at the beginning of the new century" Research evaluation, 2000, v. 8, n. 2, pp. 81-86.

Wang, Chun-Hsien; Lu, Iuan-Yuan; Chen, Chie-Bein. "Evaluating firm technological innovation capability under uncertainty". Technovation, 2008 , v. 28 , n. 6 , pp. $349-363$.

Wang, Eric C.; Huang, Weichiao. "Relative efficiency of R\&D activities: a cross-country study for environmental factors in the DEA approach". Research policy, 2007, v. 36, n. 2, pp. 260-273.

Wu, Liang-Chuan; Ong, Chorng-Shyong. "Management of information technology investment: a framework based on a real options and mean-variance theory perspective". Technovation, 2008, v. 28, n. 3, pp. 122-134.

Young, Beth; Hewitt-Dundas, Nola; Roper, Stephen. "Intellectual property management in publicly funded R\&D centres -A comparison of university-based and company-based research centres". Technovation, 2008, v. 28 , n. 8 , pp. $473-484$.

José-Luis Pino-Mejías, Universidad de Sevilla, Espa$\tilde{n} a$.

jlpino@us.es

Francisco M. Solís-Cabrera, Junta de Andalucía, España.

franciscom.solis@juntadeandalucia.es

Mercedes Delgado-Fernández, Instituto Superior Politécnico "José Antonio Echeverría" (Cujae), Cuba. mdelgado@ind.cujae.edu.cu

Rosario-del-Carmen Barea-Barrera, Universidad de Sevilla, España.

rbarea@us.es 